\title{
A Comparative Preliminary Analysis of Selected Fruit Peel Waste Fermented Solutions: Impact of Shorter Fermentation in Biosurfactant Production
}

\author{
Kamaraj Murugesan $^{1 *}$, Yabsera Tesfaye ${ }^{1}$, Afrah Mahmmud ${ }^{1}$, Esetna Tsegaye ${ }^{1}$, Tigist Getachew ${ }^{1}$, Yikerta Argaw ${ }^{1}$ \\ ${ }^{1}$ Department of Biotechnology, College of Biological and Chemical Engineering, Addis Ababa Science and Technology University, \\ Addis Ababa-16417, Ethiopia
}

Corresponding Author: Kamaraj Murugesan, PhD, Associate Professor, Department of Biotechnology, College of Biological and Chemical Engineering, Addis Ababa Science and Technology University, Addis Ababa-16417, Ethiopia. Tel: +251-966706259, Email: drkamarajm@gmail.com

Received February 20, 2019; Accepted May 16, 2019; Online Published June 20, 2019

\begin{abstract}
Introduction: Fruit waste mediated biosurfactant (BS) embraces the considerable attention in this green chemistry era to provide an environment benign application. In this study, the impact of a shorter fermentation on the BS production was studied by employing selected fruit peels as a cheaper substrate.

Materials and Methods: The avocado, banana, lemon and pineapple fruit peel wastes were collected and used for fermentation along with water and molasses. The setup was treated separately with and without yeast in order to study its effects in fermentation.

Results: The effect of yeast as a catalyst in a shorter fermentation period has been found to be negative. The emulsification index (E24) values indicated that the fermented solutions of banana and lemon have better emulsification activity compared to the other fruit waste fermented solutions produced in this study. Foam formation, color removal, and seed germination values suggested that the BS production was very minimum and alcohol was found to be dominant in all the fermented solutions.

Conclusions: It can be concluded that the fermentation periods of 30 days are not sufficient to produce the BS in higher quality and quantities by using fruit peels. This is while the fruit peels used in this study are capable to produce and can be used as renewable, eco-friendly, and economic substrates for producing BS in an appropriate fermentation period. Still, further studies are needed to elucidate the complete chemical reaction and the components involved in the experimental setup tested in this study.

Keywords: Biosurfactant, Fruit Waste, Fermentation, Emulsification, Efficiency

Citation: C. A comparative preliminary analysis of selected fruit peel waste fermented solutions: impact of shorter fermentation in biosurfactant production. J Appl Biotechnol Rep. 2019;6(2):69-72. doi:10.29252/JABR.06.02.05.
\end{abstract}

\section{Introduction}

The massive imbalance between the cultivation and consumption of food has been derived from the average of $30 \%-40 \%$ food waste around the globe. In recent years, the effect of fruit waste is identified as a major concern in the factors contributing to the global environmental burden, due to many factors. ${ }^{1}$ In developing countries, the majority of small and micro-scale industries facing the constraints to manage the fruit wastes which are generated during their operations. Thus, processed fruit waste has been considered to be economically no value compared to the fruit products. Hence, an extremely high amount of fruit waste is discarded in the environment in an informal way by fruit processing industries and also by local fruit juice shops. As this fruit waste is rich in moisture and nutrients, it favors the microbial growth, and as a result persistently leads to environmental pollution. ${ }^{1}$

In order to solve this issue, the bioconversion of these fruit wastes into value-added renewable substrates for the production of bio-actives such as BS is considered to be a major significance in the near future due to the benefits it has such as, pollution remediation, easiness and being costeffective, less energy and capital cost, gained revenue from waste, economic development, etc. ${ }^{2}$ In recent years, the BS (surface-active molecules of biological origin) has received interest compared to synthetic surfactants, because of the advantages it has such as biodegradable, less toxicity, $\mathrm{pH}$ and temperature tolerance, environmental compatibility, high foam formation, etc. ${ }^{3}$ The processing of fruit peels produces substantial volumes of by-products often containing valued compounds in their peel. These by-products pose a complex economic burden on the production and additional waste disposal problem. ${ }^{4}$

Researchers have utilized fruit peels as a supplementation in the media for the production of BS by mostly a single microorganism which is capable of producing BS. Kumar et al. ${ }^{5}$ utilized orange peel as the best substrate for Bacillus licheniformis to yield $1.796 \mathrm{~g} / \mathrm{L}$ of $\mathrm{BS}$ and emulsification

Copyright (C) 2019 The Author(s). This is an open-access article distributed under the terms of the Creative Commons Attribution License (http:// creativecommons.org/licenses/by/4.0), which permits unrestricted use, distribution, and reproduction in any medium, provided the original work is properly cited. 
activity of $75.17 \%$ against diesel. The BS producing bacteria was evaluated by Chooklin et $\mathrm{al}^{6}$ for BS production by using the banana peel as a sole carbon source. The highest BS production $(5.30 \mathrm{~g} / \mathrm{L})$ was obtained when the Halobacteriaceae archaeon AS65 cells were grown in a minimal salt medium containing $35 \%(\mathrm{w} / \mathrm{v})$ banana peel and $1 \mathrm{~g} / \mathrm{L}$ commercial monosodium glutamate at $30^{\circ} \mathrm{C}$ and $200 \mathrm{rpm}$ after 54 hours of cultivation. Rosukon from Thailand has developed a simple and inexpensive method to produce bio-cleaners by using only the natural microbial flora existing in the fruit peel waste with the addition of brown sugar and water. ${ }^{7}$ Different fruit peels such as pineapple, lemon and banana have been widely used for the production of bio-cleaners even in commercial aspects and all these benefits the outcome of 90 days fermentation. ${ }^{7}$ According to the literature review, no report has been observed as an evidence of the impact of 30 days fermentation of fruit peels such as avocado, banana, lemon, pineapple for the production of bio-cleaners/BS. Hence, the present study has aimed to explore the possibility of producing bio-cleaners/BS during 30 days of submerged fermentation of selected fruit peels. Also, the present study evaluates the preliminary emulsification and stain removal activity of fermented solutions (FS) as an indicator of BS activity.

\section{Materials and Methods}

Fruit wastes such as banana, avocado, lemon, and pineapple were collected from various fresh juice stall available in the surroundings of Addis Ababa Science and Technology University, Addis Ababa, Ethiopia. The fermentation medium was prepared in a plastic screw cap container with mashed fruit peels $(250 \mathrm{~g})$, molasses $(20 \mathrm{~mL})$ and water $(2 \mathrm{~L})$ and were allowed to ferment for 30 days in dark conditions under room temperature $\left(29 \pm 2^{\circ} \mathrm{C}\right)$. The $\mathrm{pH}$ changes in the fermented medium were measured once in a week for 30 days. The same setup was prepared for the above-mentioned fruit peels with and without $2 \mathrm{mg}$ of yeast (Saccharomyces cerevisiae). The fermented culture was filtered through a Whatman no. 1 filter paper and centrifuged at $5000 \times \mathrm{g}$ for 20 minutes and was used as crude bio-actives for further experiments. The fermented solutions of avocado, banana, lemon, and pineapple were labeled as AFS, BFS, LFS, and PFS (without yeast) and AYFS, BYFS, LYFS, and PYFS (Yeast added solution) respectively for further identification. The emulsification index (E24) of FS was determined by mixing $2 \mathrm{~mL}$ oil (olive oil, sesame oil, coconut oil, castor oil, sunflower oil, and cumin oil) with $2 \mathrm{~mL}$ of fermented solutions according to Cooper and Goldenberg. ${ }^{8}$ The foam formation of FS was observed by handshaking the crude solutions for several minutes. The stain removal activity of FS was measured by immersing the cloth spotted $(1 \mathrm{x} 1 \mathrm{~cm})$ with dye (Congo red and Methyl violet) into the FS for 2 hours and the visible color removal was recorded. The phytotoxicity of the FS was evaluated in a static test by seed germination of Mung bean (Vigna radiata) and Fenugreek (Trigonella foenu-graecum) in a petri dish containing blotting paper according to Das and Kumar. ${ }^{9}$ In all the experiments, Tween 20 and Distilled water were used as a positive and negative control, respectively. The FS was treated by acid precipitation to extract the $\mathrm{BS}$, by adding drops of $6 \mathrm{~N} \mathrm{HCl}$ until the solution reached $\mathrm{pH} 2.0$ and was stored at $4^{\circ} \mathrm{C}$ for overnight precipitation. ${ }^{10}$ All the experiments were performed in triplicates and average values were reported.

\section{Results}

The initial $\mathrm{pH}$ was observed as 5, 5.3, 4.2, and 5.4 for the fermented medium with the fruit peels such as avocado, banana, lemon, and pineapple respectively. The $\mathrm{pH}$ did not change a lot during the fermentation for the fruit samples with avocado and banana. This is while, a significant reduction of $\mathrm{pH}$ was observed in the fermentation medium of lemon and pineapple inoculated without yeast compared to the one with added yeast (Figure 1). All FS have exhibited the emulsification effects on olive oil, sesame oil, coconut oil, castor oil, sunflower oil, and cumin oil, and the E24 calculated in the range of $20 \%-67 \%$ (Figure 2). The cumulative emulsification effects of each FS against all 6 oils tested in this study have been observed as 39\%, 50\%, 49\%, 46\%, 40\%, 42\%, $53 \%$, and $48 \%$ for AFS, BFS, LFS, PFS, AYFS, BYFS, LYFS, and PYFS respectively. The foam formation was observed in all the FS by handshaking for 2 minutes. This is while, the formed foam is not stable more than 5 minutes in all the FS except, BYFS, PFS, and PYFS. The total disappearance of the

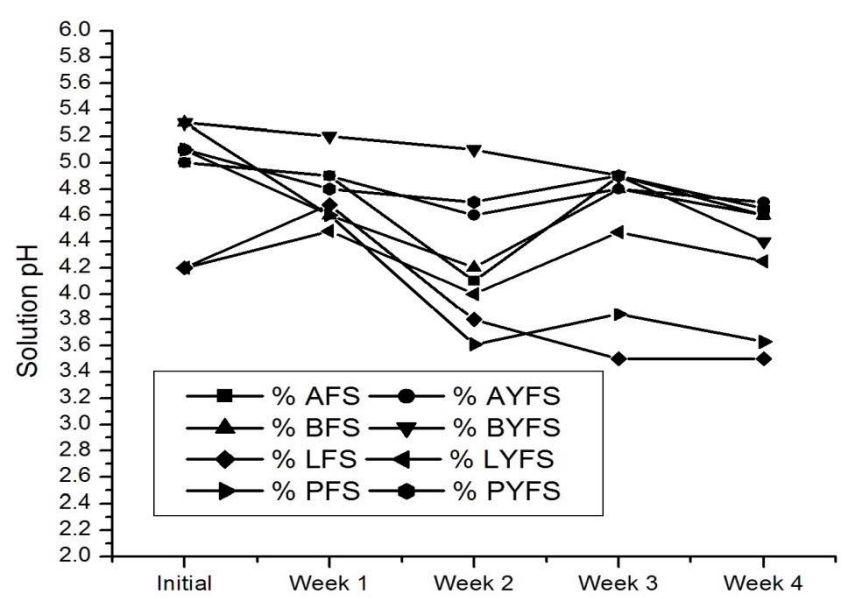

Figure 1. $\mathrm{pH}$ Variation of the Medium During Fermentation.

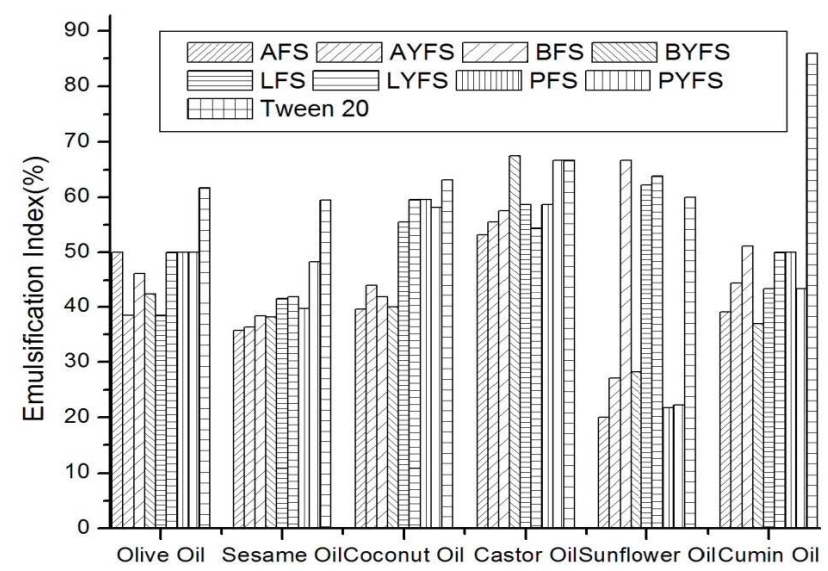

Figure 2. Emulsification Index (\%) of Fermented Solutions. 
foam in BYFS, PFS, and PYFS are observed at 10, 13 and 20 minutes respectively. These three sample results were marked as moderate activity and the other remaining sample results were reported as negative. The foam stability was disappeared in the positive control after 2 hours. The foam formation was classified as very strong, strong, moderate and negative for the tested samples (Table 1). None of the FS retained the foam for more than 1 hour which indicated the limited presence of BS molecules in the solution. The color removal efficiency of all FS was observed as moderate in cloth with Congo red and methyl violet compared with the strong activity of Tween 20 (Table 1). Seed germination (\%) was observed in the range as $16 \%-30 \%$ and $30 \%$ for FS treated and Tween 20 treated Mungbean seeds respectively. The seed germination was perceived as $0 \%$ and $20 \%$ for Fenugreek seeds treated with FS and Tween 20 respectively. All the control seeds treated with distilled water has $100 \%$ seed germination (Table 1). No precipitates were observed in the FS-treated with the acid precipitation experiment.

\section{Discussion}

Biosurfactant is an amphiphilic biological compound produced as part of the cell membrane or extracellularly by a yeast, filamentous fungi, and bacteria. It is widely used in oil, food and pharmaceutical industries due to its potential applications including emulsification, foaming, surface activity, etc. ${ }^{11}$ In recent days, bio-enzymes with BS activity are widely produced by employing fruit wastes by fermentation. In this aspect, the present study emphasized to study the possibility of producing BS in a shorter fermentation period of 30 days. Enzymes acting at alkaline $\mathrm{pH}$ are receiving the attention of detergent industries as a detergent additive. ${ }^{12}$ In this study, the $\mathrm{pH}$ value of all FS has been found to be acidic in nature, which clarifies these agents. The E24 is a vital indicator for the investigation of the emulsification activity of BS and is used to estimate the level of BS used for environmental pollution like oil recovery. ${ }^{13}$ The emulsification E24 value greater than $50 \%$ indicates positive results. In the present study, the E24 index of FS has reached the range of 39\%-49\%, except for BFS (50\%) and LFS (53\%), as an indicator of less production of BS during 30 days of fermentation. Due to high surface activity, the continuous foam formation happens in the
BS production process and it acts as an indicator for effective BS formation. ${ }^{14}$ The absence of strong foam formation and less stability observed in the FS prepared in this study is an added evidence for the absence of high BS production. Even the absence of strong BS in FS and the color removal efficiency which was found to be moderate in the cloth treated with FS, maybe due to the presence of alcohol which was produced during the fermentation of 30 days. Germination of seeds treated with FS is suggesting that the FS has failed to fulfill the characteristics of lower toxicity and higher biodegradability which is a key factor of BS application. ${ }^{15}$ Germination value of more than $80 \%$ has been considered as an indicator of the disappearance of phytotoxicity. ${ }^{16}$ The obtained results (16\%$30 \%)$ in this study indicated that the FS showed inhibitory effects on seeds. In addition, no precipitates were produced in a FS after $24 \mathrm{~h}$ incubation of acid precipitation, which supports the no production of sufficient BS in all FS at 30 days fermentation. The profile of individual cell growth was not given consideration due to the presence of various microorganisms in FS at earlier stages. It is known that the presence of other microbes will be reduced from the first week to fourth week, due to the formation of alcohol in the FS. It was observed that only yeast cells are predominantly present in the later stages of fermentation. ${ }^{17}$ Moreover, the main objective of using the yeast strain in one set of experiments was to compare the efficiency of yeast as a catalyst to enhance the fermentation process. Since no difference is found in the activity of FS with and without yeast during the 30 day fermentation period, further research has not been carried out on biomass content.

\section{Conclusions}

The present study was centered on a 30-day fermentation experiment of selected fruit peel waste with and without yeast to assess the possibility of BS production. The results of a series, preliminary analysis, such as $\mathrm{pH}$, Emulsification activity, foam formation and stability, color removal and seed germination experiments yielded useful information regarding the production possibility of BS in a 30 day fermentation period. It can be concluded that the 30 days fermentation is not satisfactory and has failed to produce a more amount of BS with an efficient activity. Still, the peels of

Table 1. Biosurfactant Characteristics of Fermented Solutions

\begin{tabular}{|c|c|c|c|c|c|c|}
\hline \multirow{3}{*}{ Sample } & \multicolumn{6}{|c|}{ Biosurfactant activity } \\
\hline & \multicolumn{2}{|c|}{ Foam Characteristics } & \multicolumn{2}{|c|}{ Color Removal Efficiency From Cloth } & \multicolumn{2}{|c|}{ Seed Germination (\%) } \\
\hline & Foam Formation & Foam Stability & Congo Red & Methyl Violet & Mung Bean Seed & Fenugreek Seed \\
\hline AFS & + & - & + & + & 30 & - \\
\hline AYFS & + & - & + & + & 16 & - \\
\hline BFS & + & - & + & + & 16 & - \\
\hline BYFS & ++ & + & + & + & 16 & - \\
\hline LFS & + & - & + & + & 16 & - \\
\hline LYFS & + & - & + & + & 16 & - \\
\hline PFS & ++ & + & + & + & 25 & - \\
\hline PYFS & ++ & + & + & + & 16 & - \\
\hline Tween 20 & ++ & ++ & ++ & ++ & 30 & 20 \\
\hline Distilled Water & - & - & - & - & 100 & 100 \\
\hline
\end{tabular}

(++ Strong; + Moderate; - Negative). 
fruits such as avocado, banana, lemon, and pineapple can be effectively used as a substrate for a successful production of BS in an extended/sufficient period of fermentation.

\section{Authors' Contributions}

All authors contributed equally to this research.

\section{Conflict of Interest Disclosures}

The authors declare they have no conflicts of interest.

\section{Acknowledgments}

The authors are thankful to the Addis Ababa Science and Technology University, Ethiopia for providing financial support (Student Research Grant Reference No-AASTU-SSRG-CBCE-DBT-1-2019) and lab facilities for this research.

\section{References}

1. Banerjee J, Singh R, Vijayaraghavan R, MacFarlane D, Patti AF, Arora A. Bioactives from fruit processing wastes: Green approaches to valuable chemicals. Food Chem. 2017;225:10-22. doi:10.1016/j. foodchem.2016.12.093.

2. Banat IM, Satpute SK, Cameotra SS, Patil R, Nyayanit NV. Cost effective technologies and renewable substrates for biosurfactants' production. Front Microbiol. 2014;5:697. doi:10.3389/ fmicb.2014.00697.

3. Satpute SK, Płaza GA, Banpurkar AG. Biosurfactants' production from renewable natural resources: example of innovativeand smart technology in circular bioeconomy. Manag Syst Prod Eng. 2017;25(1):46-54. doi:10.1515/mspe-2017-0007.

4. Putnik P, Bursac Kovacevic D, Rezek Jambrak A, et al. Innovative "green" and novel strategies for the extraction of bioactive added value compounds from citrus wastes-a review. Molecules. 2017;22(5). doi:10.3390/molecules22050680.

5. Kumar AP, Janardhan A, Viswanath B, Monika K, Jung JY, Narasimha G. Evaluation of orange peel for biosurfactant production by Bacillus licheniformis and their ability to degrade naphthalene and crude oil. 3 Biotech. 2016;6(1):43. doi:10.1007/s13205-0150362-x.

6. Chooklin CS, Maneerat S, Saimmai A. Utilization of banana peel as a novel substrate for biosurfactant production by Halobacteriaceae archaeon AS65. Appl Biochem Biotechnol. 2014;173(2):624-645. doi:10.1007/s12010-014-0870-x.
7. Thirumurugan P, Mathivanan K. Production and analysis of enzyme bio-cleaners from fruit and vegetable wastes by using yeast and bacteria. Student project Report (D.O.Rc.No.1082/2015A; Project No: 28) submitted to Tamil Nadu State Council for Higher Education (TANSCHE), India; 2016. p. 4-6.

8. Cooper DG, Goldenberg BG. Surface-active agents from two bacillus species. Appl Environ Microbiol. 1987;53(2):224-229.

9. Das AJ, Kumar R. Utilization of agro-industrial waste for biosurfactant production under submerged fermentation and its application in oil recovery from sand matrix. Bioresour Technol. 2018;260:233-240. doi:10.1016/j.biortech.2018.03.093.

10. Das P, Mukherjee S, Sen R. Antimicrobial potential of a lipopeptide biosurfactant derived from a marine Bacillus circulans. J Appl Microbiol. 2008;104(6):1675-1684. doi:10.1111/j.13652672.2007.03701.x.

11. Suresh Chander CR, Lohitnath T, Mukesh Kumar DJ, Kalaichelvan PT. Production and characterization of biosurfactant from Bacillus subtilis MTCC441 and its evaluation to use as bioemulsifier for food bio-preservative. Adv Appl Sci Res. 2012;3(3):1827-1831.

12. Anwar A, Saleemuddin M. Alkaline proteases: A review. Bioresour Technol. 1998;64(3):175-183. doi:10.1016/S09608524(97)00182-X.

13. Sarubbo LA, Rufino RD, Luna JM. Application of a biosurfactant produced in low-cost substrates in the removal of hydrophobic contaminants. Chem Eng Trans. 2015;43:295-300. doi:10.3303/ CET1543050.

14. Winterburn JB, Russell AB, Martin PJ. Integrated recirculating foam fractionation for the continuous recovery of biosurfactant from fermenters. Biochem Eng J. 2011;54(2):132-139. doi:10.1016/j. bej.2011.02.011.

15. Franzetti A, Gandolfi I, Bestetti G, Banat IM. (Bio) surfactant and bioremediation, successes and failures. In: Płaza G, ed. Trends in Bioremediation and Phytoremediation. Kerala, India: Research Signpost; 2010:145-156.

16. Rufino RD, de Luna JM, de Campos Takaki GM, Sarubbo LA. Characterization and properties of the biosurfactant produced by Candida lipolytica UCP 0988. Electron J Biotechnol. 2014;17(1):34-38. doi:10.1016/j.ejbt.2013.12.006.

17. Nasir A, Rahman SS, Hossain MM, Choudhury N. Isolation of Saccharomyces cerevisiae from pineapple and orange and study of metal's effectiveness on ethanol production. Eur J Microbiol Immunol (Bp). 2017;7(1):76-91. doi:10.1556/1886.2016.00035 